\title{
Alteration of extracellular superoxide dismutase expression is associated with an aggressive phenotype of oral squamous-cell carcinoma
}

\author{
HIDETAKA YOKOE $^{1 *}$, HITOMI NOMURA $^{2 *}$, YUKIO YAMANO ${ }^{2}$, KAZUAKI FUSHIMI $^{2}$, \\ YOSUKE SAKAMOTO $^{1}$, KATSUNORI OGAWARA ${ }^{2}$, MASASHI SHIIBA ${ }^{2}$, HIROKI BUKAWA $^{2}$, \\ KATSUHIRO UZAWA $^{1,2}$, YUICHI TAKIGUCHI ${ }^{3}$ and HIDEKI TANZAWA ${ }^{1,2}$ \\ ${ }^{1}$ Division of Dentistry and Oral-Maxillofacial Surgery, Chiba University Hospital; Departments of \\ ${ }^{2}$ Clinical Molecular Biology, and ${ }^{3}$ Respirology, Graduate School of Medicine, Chiba University, Chiba 260-8670, Japan
}

Received February 17, 2010; Accepted March 25, 2010

DOI: 10.3892/etm_00000092

\begin{abstract}
Oxidative stress results in damage to cellular structures and has been linked to numerous diseases, including cancer. Extracellular superoxide dismutase (EC-SOD) is a principal enzymatic antioxidant in extracellular space. The purpose of this study was to determine whether the expression of EC-SOD protein is altered in the carcinogenetic process of oral squamous-cell carcinoma (OSCC). Immunohistochemical analysis was carried out in matched normal and tumour specimens collected from 58 OSCCs and 20 oral premalignant lesions (OPLs). Correlations between the EC-SOD expression levels and clinicopathological features of OSCC patients were evaluated by Fisher's exact test. Although EC-SOD protein was consistently expressed on the plasma membrane of cells in normal tissues, plasma membranous EC-SOD expression was lost in almost all the OSCC specimens examined (98\%). Instead, positive EC-SOD expression was detected in the cytoplasmic compartments of cancerous cells in both OPLs (65\%) and OSCCs (52\%), together with a high incidence of lymph node metastasis $(\mathrm{p}=0.0397)$. These results suggest that the dysregulation of EC-SOD protein expression is a frequently occuring and early event in oral carcinogenesis, and that cytoplasmic EC-SOD may contribute to the increased aggressiveness of OSCC.
\end{abstract}

Correspondence to: Dr Hideki Tanzawa, Department of Clinical Molecular Biology, Graduate School of Medicine, Chiba University, 1-8-1 Inohana, Chuo-ku, Chiba 260-8670, Japan

E-mail: tanzawap@faculty.chiba-u.jp

${ }^{*}$ Contributed equally

Key words: extracellular superoxide dismutase, oral squamouscell carcinoma, immunohistochemistry, lymph node metastasis, aggressive phenotype

\section{Introduction}

Oral squamous-cell carcinoma (OSCC) is a major cause of morbidity and mortality worldwide, accounting for 275,000 new cases and more than 120,000 deaths annually (1). Despite therapeutic and diagnostic advances, patients are often diagnosed at advanced stages, and mortality rates are still increasing (2). This highlights the need for continued efforts to discover suitable biomarkers for early disease diagnosis and for the improved understanding of disease pathogenesis as a first step towards improving treatment. Considering these issues, it is imperative to study OSCC at the genetic level and to characterize the genetic changes responsible for carcinogenesis and tumour behavior.

Since cancer has the specific potential of rapid and unlimited growth, oxidative stress, which is characterized by an imbalance between the presence of relatively high levels of toxic reactive species principally consisting of reactive oxygen species (ROS) and antioxidative defense mechanisms, is a common feature in a wide range of solid tumors, including OSCC $(3,4)$. Some studies have found that cells undergoing neoplastic transformation show marked changes in metabolism, resulting in an increase in oxidative stress in cancerous cells $(5,6)$. Enhanced oxidizing status in transforming cells is thought to induce DNA damage, leading to genetic lesions that initiate tumorigenicity and facilitate immortalization, enhance cell proliferation and sustain subsequent local and systemic tumor progression (7-9). Additionally, recent studies revealed that oxidative stress is not merely toxic due to ROS production by metabolism, but also serves an important regulatory role in numerous oncogenic signaling pathways in cancerous cells $(10,11)$.

Superoxide dismutase (SOD) is generally regarded as one of the first lines of antioxidant defense in aerobic cells (12). This enzyme is highly efficient in protecting cells and tissues against oxidative stress based on the potency of the cellular defense mechanism against ROS, including true free radicals, superoxide anion $\left(\mathrm{O}_{2}^{-}\right)$and hydroxyl radical $\left(\mathrm{OH}^{-}\right)$, as well as non-radical compounds, such as hydrogen peroxide $\left(\mathrm{H}_{2} \mathrm{O}_{2}\right)$ (13). Substantial studies have reported that the effects of SOD 
Table I. Correlation between cytoplasmic EC-SOD protein expression and clinical calssification in OSCCs ( $\mathrm{n}=58)$ and OPLs $(n=20)$.

\begin{tabular}{|c|c|c|c|c|}
\hline \multirow[t]{2}{*}{ Clinical classification } & \multirow[t]{2}{*}{ Total } & \multicolumn{2}{|c|}{ Result of immunostaining: no. of patients (\%) } & \multirow[t]{2}{*}{ p-value ${ }^{a}$} \\
\hline & & Cytoplasmic EC-SOD (-) & Cytoplasmic EC-SOD (+) & \\
\hline \multicolumn{5}{|l|}{ Age at surgery (years) } \\
\hline$<60$ & 16 & $8(50)$ & $8(50)$ & \multirow{3}{*}{0.9471} \\
\hline $60-69$ & 20 & $9(45)$ & $11(55)$ & \\
\hline$\geq 70$ & 22 & $11(50)$ & $11(50)$ & \\
\hline \multicolumn{5}{|l|}{ Gender } \\
\hline Male & 44 & $19(43)$ & $25(57)$ & \multirow[t]{2}{*}{0.2244} \\
\hline Female & 14 & $9(64)$ & $5(36)$ & \\
\hline \multicolumn{5}{|l|}{ T-primary tumor } \\
\hline $\mathrm{T} 1$ & 5 & $4(80)$ & $1(20)$ & \multirow{4}{*}{0.5393} \\
\hline $\mathrm{T} 2$ & 22 & $10(45)$ & $12(55)$ & \\
\hline T3 & 14 & $7(50)$ & $7(50)$ & \\
\hline $\mathrm{T} 4$ & 17 & $7(41)$ & $10(59)$ & \\
\hline \multicolumn{5}{|l|}{ N-regional lymph node } \\
\hline $\mathrm{N}(-)$ & 31 & $19(61)$ & $12(39)$ & \multirow[t]{2}{*}{$0.0397^{\mathrm{a}}$} \\
\hline $\mathrm{N}(+)$ & 27 & $9(33)$ & $18(67)$ & \\
\hline \multicolumn{5}{|l|}{ Stage } \\
\hline I & 5 & $4(80)$ & $1(20)$ & \multirow{4}{*}{0.1896} \\
\hline II & 10 & $4(40)$ & $6(60)$ & \\
\hline III & 12 & $8(67)$ & $4(33)$ & \\
\hline IV & 31 & $12(39)$ & $19(61)$ & \\
\hline \multicolumn{5}{|l|}{ Histopathological type } \\
\hline Well differentiated & 38 & $19(50)$ & $19(50)$ & \multirow[t]{2}{*}{0.7866} \\
\hline Moderately/poorly differentiated & 20 & $9(45)$ & $11(55)$ & \\
\hline \multicolumn{5}{|l|}{ Tumor site } \\
\hline Tongue & 32 & $16(50)$ & $16(50)$ & \multirow{5}{*}{0.4375} \\
\hline Gingiva & 16 & $7(44)$ & $9(56)$ & \\
\hline Oral floor & 3 & $2(67)$ & $1(33)$ & \\
\hline Buccal mucosa & 6 & $2(33)$ & $4(67)$ & \\
\hline Lip & 1 & $1(100)$ & $0(0)$ & \\
\hline \multicolumn{5}{|l|}{ Leukoplakia } \\
\hline Mild dysplasia & 2 & $1(50)$ & $1(50)$ & \multirow{3}{*}{0.3981} \\
\hline Moderate dysplasia & 11 & $5(45)$ & $6(55)$ & \\
\hline Severe dysplasia & 7 & $1(14)$ & $6(86)$ & \\
\hline
\end{tabular}

aProbability of $\mathrm{p}<0.05$.

in human malignancies are associated with tumor growth and drug resistance in vitro and in vivo $(14,15)$. To date, three distinct isoforms of SOD and their distribution have been characterized in mammals (16). Of these, extracellular superoxide dismutase (EC-SOD) is the only isoform that is mainly expressed in the extracellular space via binding with heparin sulfate proteoglycans (17). Since the extracellular space is known to have many potential sources of ROS and to be a relatively more oxidized state than the interior of cells, dysregulation of extracellular oxidant-moderating proteins, including EC-SOD, is considered more important in cancer (18-20). However, in contrast to the intracellular SODs, little is known regarding EC-SOD in human tumors, including
OSCC. The purpose of the present study was therefore to determine EC-SOD protein expression in a series of human primary OSCCs and oral premalignant lesions (OPLs), and to correlate the expression with its clinical relevance in patients with OSCC.

\section{Materials and methods}

Tissue specimens. Fifty-eight pairs of primary OSCC samples and corresponding normal oral epithelium tissues were obtained at the time of surgery, performed at Chiba University Hospital between 1998 and 2007. All patients provided their informed consent according to the study protocol, which was 

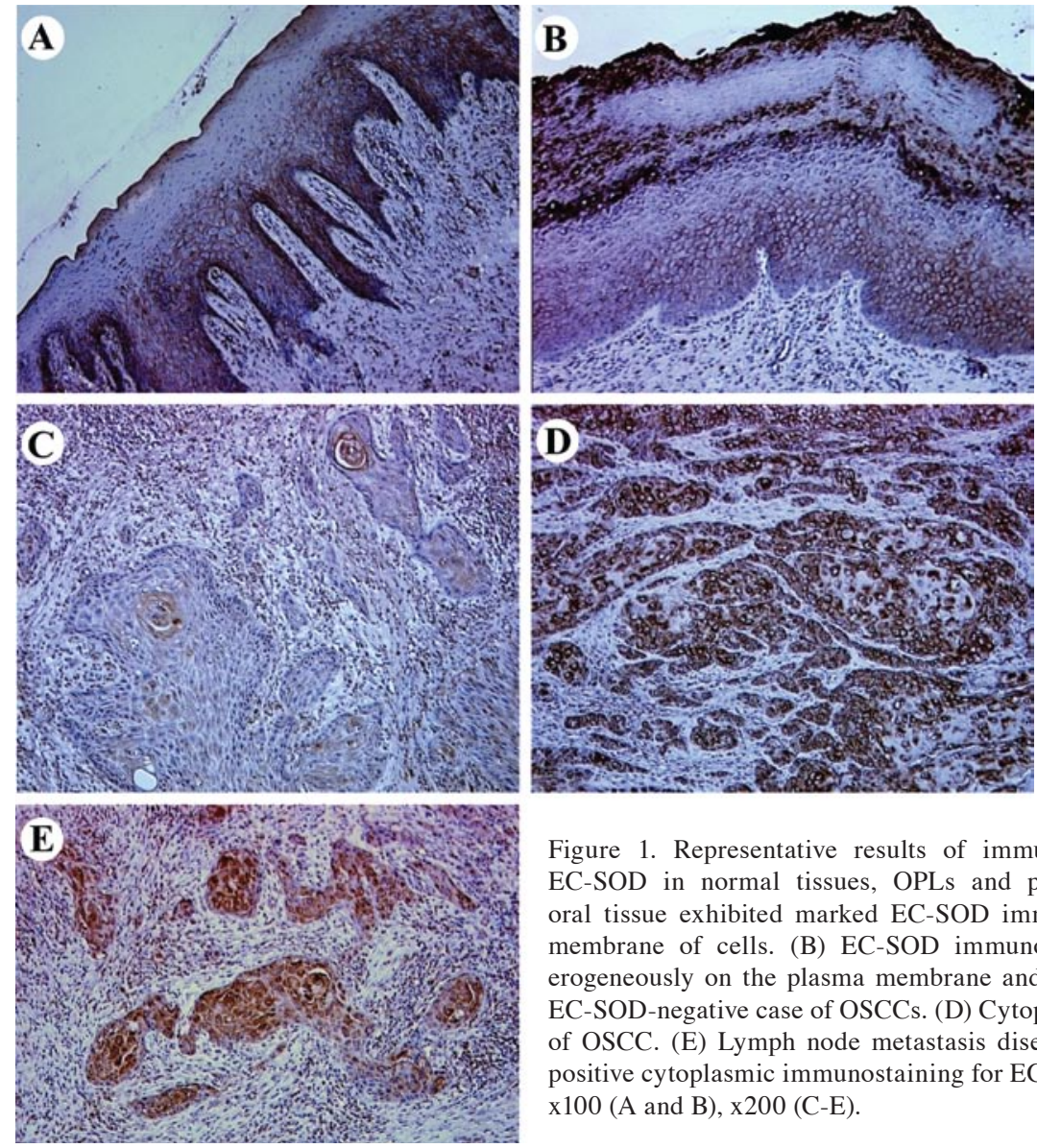

Figure 1. Representative results of immunohistochemical staining of EC-SOD in normal tissues, OPLs and primary OSCCs. (A) Normal oral tissue exhibited marked EC-SOD immunoreactivity on the plasma membrane of cells. (B) EC-SOD immunoreactivity was detected heterogeneously on the plasma membrane and cytoplasm of OPL cells. (C) EC-SOD-negative case of OSCCs. (D) Cytoplasmic EC-SOD-positive case of OSCC. (E) Lymph node metastasis disease specimens also exhibited positive cytoplasmic immunostaining for EC-SOD. Original magnification x100 (A and B), x200 (C-E).

reviewed and approved by the institutional review board of Chiba University before any procedures were performed. In addition, 20 samples from cases of advanced OPLs pathologically diagnosed as leukoplakia with epithelial dysplasia, i.e., mild $(n=2)$, moderate $(n=11)$ and severe $(n=7)$, in a high-risk oral site, such as the ventral-lateral tongue or gingiva, were obtained as described above.

Histopathological diagnosis of each tumor specimen was performed according to the International Histological Classification of Tumors by the Department of Pathology, Chiba University Hospital. Clinicopathological staging was determined by the TNM classification of the International Union against Cancer.

Immunohistochemistry. Immunohistochemical (IHC) staining was carried out on 4- $\mu$ m sections of paraffin-embedded specimens. The clinicopathological characteristics of the OSCCs in this series are summarized in Table I. Briefly, after deparaffinization and hydration, the slides were pre-treated in $10 \mathrm{mM}$ sodium citrate buffer ( $\mathrm{pH} 6.0$ ) in a microwave oven for $5 \mathrm{~min}$ at $95^{\circ} \mathrm{C}$. Endogenous peroxidase activity was quenched by a 30 -min incubation in a mixture of $0.3 \%$ hydrogen peroxide solution in $100 \%$ methanol. After being washed with PBS buffer, the sections were incubated with primary antibody affinity-purified goat antihuman EC-SOD polyclonal antibody (1:100 dilution; Santa Cruz Biotechnology) at room temperature in a moist chamber for $2 \mathrm{~h}$. After being washed with PBS buffer, the slides were treated with peroxidase-labeled secondary antibody for $1 \mathrm{~h}$ followed by color development in 3,3'-diaminobenzidine tetrahydrochloride (Dako Japan Inc.). Finally, the slides were lightly counterstained with hematoxylin. As negative controls, the slides were incubated with PBS instead of primary antibodies. To quantitate the state of EC-SOD protein expression in these of components, we used previously described Histo $(\mathrm{H})$-score systems $(21,22)$. In brief, the mean percentage of the epithelial cells that showed a persistent EC-SOD signal was respectively determined in at least five distinct fields in each section at a magnification of $x 400$. The intensity of the immunoreaction was scored as follows: $1^{+}$, weak; $2^{+}$, moderate; $3^{+}$, intense. Three target cell types, normal, pre-malignant and malignant epithelial cells, were identified for scoring. The percentage of EC-SODpositive cells and the staining intensity were then multiplied to produce an EC-SOD H-score. Cases with a cytoplasmic EC-SOD H-score exceeding 50.15 (maximum score of normal tissues) were considered positive. Specimens were evaluated by two independent pathologists, neither of whom had knowledge of patient clinical status.

Statistical analysis. Statistical analysis of the OSCC tissue and corresponding normal tissue was performed using Wilcoxon's signed rank sum test. The comparison OPLs and other tissue including normal samples and OSCCs or the Mn-SOD-positive and Mn-SOD-negative cases of OSCC were analyzed using the Mann-Whitney U-test. Correlations between Mn-SOD $\mathrm{H}$-score and clinicopathological features were evaluated by Fisher's exact test. Data are expressed as the median values. $p<0.05$ was considered statistically significant. 
A

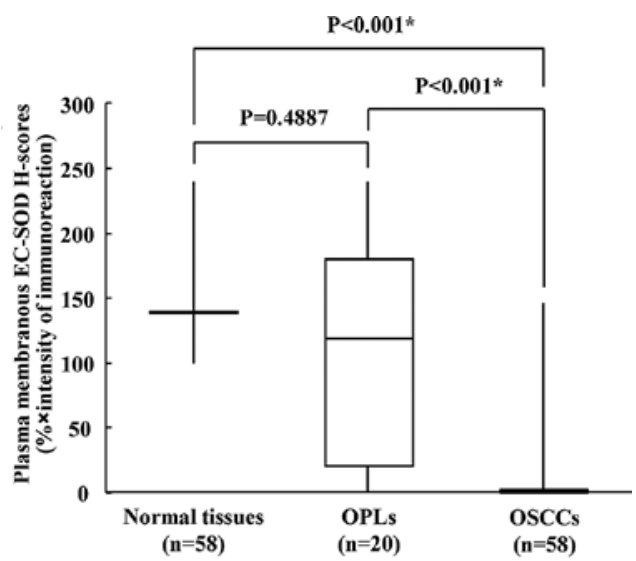

B

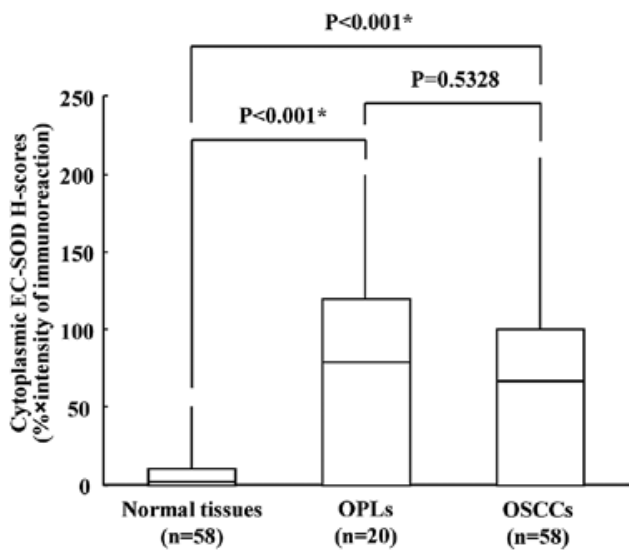

Figure 2. The state of EC-SOD protein expression in normal tissues $(n=58)$, OPLs $(n=20)$ and primary OSCCs $(n=58)$. (A) H-scores for plasma membranous EC-SOD protein expression. Plasma membranous EC-SOD protein expression was significantly down-regulated in OSCCs compared to normal oral tissue $(\mathrm{p}<0.001$, Wilcoxon's signed rank sum test) and OPLs $(\mathrm{p}<0.001$, MannWhitney U-test). (B) H-scores for cytoplasmic EC-SOD protein expression. The protein expression levels of cytoplasmic EC-SOD in OSCCs $(\mathrm{p}<0.001$, Wilcoxon's signed rank sum test) and OPLs ( $\mathrm{p}<0.001$, Mann-Whitney U-test) were significantly elevated compared to those in normal compartments. Results are expressed as the mean $\pm \mathrm{SD}$. H-score, histo-score; ${ }^{*} \mathrm{p}<0.05$.

\section{Results}

EC-SOD expression in OSCCs and OPLs. IHC staining was performed using a series of OSCC specimens, including 58 OSCCs with corresponding normal tissues and 20 OPLs that were histopathologically diagnosed as leukoplakia with epithelial dysplasia. Considering that the evidence indicated that the malignant transformation rate of oral leukoplakia with dysplasia was apparently higher than that of oral leukoplakia without dysplasia (23), patients with advanced OPLs, defined as leukoplakia exhibiting epithelial dysplasia, were eligible for the trial. Representative results for EC-SOD protein expression in normal oral tissues, OPLs and primary OSCCs are shown in Fig. 1.

Normal oral mucosal specimens exhibited consistently strong EC-SOD immunoreaction on the plasma membrane of cells. In OPLs, a loss of plasma membranous EC-SOD immunostaining was observed in some cases, but many of the specimens revealed positive immunoreactivity for EC-SOD on the plasma membrane of cells. Notably, positive staining of the protein was also detected on the cytoplasm of OPL cells in

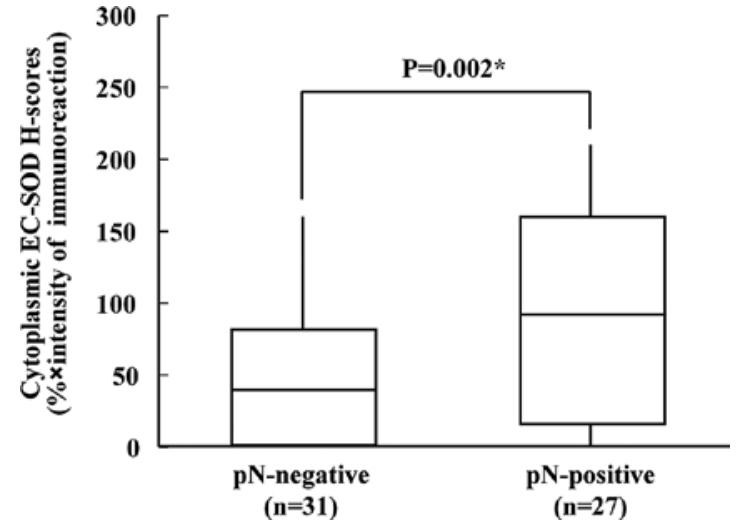

Figure 3. $\mathrm{H}$-scores for node-negative $(\mathrm{n}=31)$ vs. node-positive cases $(\mathrm{n}=27)$ compared. EC-SOD protein expression levels were significantly higher in $\mathrm{pN}$-positive OSCC tissue compared to those in $\mathrm{pN}$-negative tissue $(\mathrm{p}=0.002$, Mann-Whitney U-test). Results are expressed as the mean $\pm \mathrm{SD}$. H-score, histo-score; " $\mathrm{p}<0.05$.

$65 \%$ of the specimens examined. Regarding OSCCs, plasma membranous EC-SOD immunoreaction was largely lost in the specimens examined (98\%), whereas strong immunoreactivity of the protein was observed on the cytoplasm of cancerous cells in $52 \%$ of the specimens (cytoplasmic EC-SODpositive). According to the $\mathrm{H}$-scores, the expression levels of plasma membrane EC-SOD were significantly reduced in OSCCs compared to their normal counterparts (Wilcoxon's signed rank sum test, $\mathrm{p}<0.001$; Fig. 2A), whereas cytoplasmic EC-SOD expression levels were considerably higher not only in OSCCs (Wilcoxon's signed rank sum test, $\mathrm{p}<0.001$; Fig. 2B), but also in OPLs (Mann-Whitney U-test, $\mathrm{p}<0.001$ ) compared to normal tissues.

Correlation of EC-SOD expression with clinicopathological parameters. In the present study, $52 \%$ of the OSCC patients were characterized as EC-SOD-positive in the cytoplasmic compartments of cancerous cells. The correlation between the clinicopathological characteristics of the patients with OSCC and the status of cytoplasmic EC-SOD expression is summarized in Table I. Positive EC-SOD expression of cytoplasm was significantly associated with regional lymph node metastasis (Fisher's exact test, $\mathrm{p}=0.0397$ ). The cytoplasmic EC-SOD H-scores for tumors with lymph node metastasis and for those without ranged from 0 to 210.55 (median 100.03) and from 0 to 160.02 (median 30.47), respectively. Cytoplasmic EC-SOD expression levels were significantly higher in patients with OSCC who were pN-positive, compared to those who were $\mathrm{pN}$-negative (Mann-Whitney U-test, $\mathrm{p}=0.002$; Fig. 3).

\section{Disscussion}

In the present study, we initially characterized EC-SOD protein expression in cancerous and pre-cancerous lesions of the oral cavity using IHC analysis. Of particular interest is that, although normal oral epithelium tissues consistently exhibited positive expression of EC-SOD on the plasma membrane of cells in all specimens, plasma membranous EC-SOD expression was significantly down-regulated in almost all the OSCC specimens examined (98\%), indicating that EC-SOD may be 
critical for normal functioning, and that the loss of the protein resultsan increased risk of cancer development in cells. This notion is supported by a number of previous studies in vitro and in vivo. Kim et al demonstrated that EC-SOD transgenic mice showed reduced incidence of tumors induced by dimethylbenzanthracene/12-O-tetradecanoylphorbol-13acetate compared to controls (24). EC-SOD overexpression significantly inhibited the growth of B16 melanomas with high growth potential and decreased the metastatic behavior of lung cancer cells in mice (25). Moreover, a recent study revealed that EC-SOD inhibited the invasive capacity of human prostate cancer cells. This was correlated with reduced metalloproteinase activities (26), suggesting that EC-SOD may have the potential to suppress aggressive tumor behavior.

Conversely, our study also identified altered localization of EC-SOD protein at a high level of expression on the cytoplasm of cancerous cells in 52\% of the OSCC specimens examined $(\mathrm{p}<0.001)$. Additionally, cytoplasmic EC-SOD overexpression was associated with an aggressive phenotype of OSCC, including lymph node metastasis $(\mathrm{p}=0.0397)$. The exact cause of the heterogeneous distribution of EC-SOD in cancerous cells is not as yet clear; however, the most likely cause is the polymorphic variant of the EC-SOD gene, which involves a single nucleotide substitution ( $\mathrm{G}$ to $\mathrm{C}$ ) and results in an amino acid change from arginine (Arg) to glycine (Gly) at 213. This amino acid substitution decreases the anchoring of EC-SOD to negatively charged polysaccharides, including heparin (27). Considering that EC-SOD localizes in extracellular spaces via binding with heparin sulfate proteoglycans, this could be linked to the EC-SOD heterogeneous localization in OSCC cells.

Subcellular localization of a protein often provides important clues to its function (28), and the transition from the benign state to the fully malignant one may involve a change in subcellular presence. Some insight into the cytoplasmic mechanism of EC-SOD associated with tumor aggressiveness can be gained from the recent findings that manganese SOD, an intracellular SOD isoform, enhances the invasive and migratory activity of tumor cells though $\mathrm{H}_{2} \mathrm{O}_{2}$ production (29), and that overexpression of the protein has been identified in various cancers together with an association with poor prognosis $(30,31)$. Recently, we also found that manganese SOD up-regulation was correlated with a high incidence of lymph node metastasis in OSCC patients (32). Together, it is possible that EC-SOD may function independently in cellular components in cancer cells, acting as a tumor-suppressor on the plasma membrane, but as a tumor-activator in the cytoplasm. Changes in cytoplasmic EC-SOD protein expression were even detected in the OPLs examined (65\%), suggesting that the dysregulation of the protein expression is a frequent and early event during oral carcinogenesis.

In conclusion, our study provides the first documentation that EC-SOD protein expression and subcellular distribution is altered in both OPLs and OSCCs, and that positive cytoplasmic EC-SOD expression is associated with an aggressive tumor phenotype in OSCCs. The molecular mechanisms linked to the aberrant expression of EC-SOD in OSCC cells remain unclear, and further studies are warranted to elucidate the molecular alterations involved in EC-SOD dysregulation in oral carcinogenesis.

\section{Acknowledgements}

This study was partly supported by a Grant-in-Aid for Scientific Research (No. 20791492) from the Ministry of Education, Culture, Sports, Science and Technology of Japan, and the Global COE Program (Global Center for Education and Research in Immune System Regulation and Treatment), MEXT, Japan.

\section{References}

1. Ferlay J, Bray P, Pisani P and Parkin DM: GLOBOCAN 2002: Cancer Incidence, Mortalityand Prevalence Worldwide. IARC Cancer Base No. 5, version 2.0. IARC Press, Lyon, 2004.

2. La Vecchia C, Lucchini F, Negri E and Levi F: Trends in oral cancer mortality in Europe. Oral Oncol 40: 433-439, 2004.

3. Valko M, Rhodes CJ, Moncol J, Izakovic M and Mazur M: Free radicals, metals and antioxidants in oxidative stress-induced cancer. Chem Biol Interact 160: 1-40, 2006.

4. Klaunig JE and Kamendulis LM: The role of oxidative stress in carcinogenesis. Annu Rev Pharmacol Toxicol 44: 239-267, 2004.

5. Oberley LW, Oberley TD and Buettner GR: Cell division in normal and transformed cells: the possible role of superoxide and hydrogen peroxide. Med Hypoth 7: 21-42, 1981.

6. Spitz DS, Sim JE, Ridnour LA, Galoforo SS and Lee YJ: Glucose deprivation-induced oxidative stress in human tumor cells: a fundamental defect in metabolism? Ann NY Acad Sci 899: 349-362, 2000.

7. Valko M, Izakovic M, Mazur M, Rhodes CJ and Telser J: Role of oxygen radicals in DNA damage and cancer incidence. Mol Cell Biochem 266: 37-56, 2004.

8. Franco R, Schoneveld O, Georgakilas AG and Panayiotidis MI: Oxidative stress, DNA methylation and carcinogenesis. Cancer Lett 266: 6-11, 2008.

9. Gromadzińska $\mathbf{J}$ and Wasowicz $\mathrm{W}$ : The role of reactive oxygen species in the development of malignancies. Int J Occup Med Environ Health 13: 233-245, 2000.

10. Kuznetsov AV, Smigelskaite J, Doblander C, et al: Survival signaling by C-RAF: mitochondrial reactive oxygen species and Ca2+ are critical targets. Mol Cell Biol 28: 2304-2313, 2008.

11. Cho HJ, Jeong HG, Lee JS, et al: Oncogenic H-Ras enhances DNA repair through the Ras/phosphatidylinositol 3-kinase/Rac1 pathway in NIH3T3 cells. Evidence for association with reactive oxygen species. J Biol Chem 277: 19358-19366, 2002.

12. Blokhina O, Virolainen E and Fagerstedt KV: Antioxidants, oxidative damage and oxygen deprivation stress: a review. Ann Bot 91: 179-194, 2003.

13. Liochev SI and Fridovich I: The effects of superoxide dismutase on $\mathrm{H} 2 \mathrm{O} 2$ formation. Free Radic Biol Med 42: 1465-1469, 2007.

14. Hileman EA, Achanta G and Huang P: Superoxide dismutase: an emerging target for cancer therapeutics. Expert Opin Ther Targets 5: 697-710, 2001.

15. Kinnula VL and Crapo JD: Superoxide dismutases in malignant cells and human tumors. Free Radic Biol Med 36: 718-744, 2004.

16. Zelko IN, Mariani TJ and Folz RJ: Superoxide dismutase multigene family: a comparison of the CuZn-SOD (SOD1), Mn-SOD (SOD2), and EC-SOD (SOD3) gene structures, evolution and expression. Free Radic Biol Med 33: 337-349, 2002.

17. Sandström J, Karlsson K, Edlund T and Marklund SL: Heparinaffinity patterns and composition of extracellular superoxide dismutase in human plasma and tissues. Biochem J 294: 853-857, 1993.

18. Moriarty-Craige SE and Jones DP: Extracellular thiols and thiol/ disulfide redox in metabolism. Annu Rev Nutr 24: 481-509, 2004.

19. Nakamura H, Masutani H and Yodoi J: Extracellular thioredoxin and thioredoxin-binding protein 2 in control of cancer. Semin Cancer Biol 16: 444-451, 2006.

20. Rees MD, Kennett EC, Whitelock JM and Davies MJ: Oxidative damage to extracellular matrix and its role in human pathologies. Free Radic Biol Med 44: 1973-2001, 2008.

21. Bilalovic N, Sandstad B, Golouh R, et al: CD10 protein expression in tumor and stromal cells of malignant melanoma is associated with tumor progression. Mod Pathol 17: 1251-1258, 2004. 
22. McCarty KS Jr, Szabo E, Flowers JL, et al: Use of monoclonal anti-estrogen receptor antibody in the immunohistochemical evaluation of human tumors. Cancer Res 46: 4244-4248, 1986.

23. Amagasa T, Yamashiro $M$ and Ishikawa $\mathrm{H}$ : Oral leukoplakia related to malignant transformation. Oral Sci Int 3: 45-55, 2006.

24. Kim SH, Kim MO, Gao P, et al: Overexpression of extracellular superoxide dismutase (EC-SOD) in mouse skin plays a protective role in DMBA/TPA-induced tumor formation. Oncol Res 15 333-341, 2005.

25. Wheeler MD, Smutney OM and Samulski RJ: Secretion of extracellular superoxide dismutase from muscle transduced with recombinant adenovirus inhibits the growth of B16 melanomas in mice. Mol Cancer Res 1: 871-881, 2003.

26. Chaiswing L, Zhong W, Cullen JJ, Oberley LW and Oberley TD: Extracellular redox state regulates features associated with prostate cancer cell invasion. Cancer Res 68: 5820-5826, 2008.

27. Sandström J, Nilsson P, Karlsson K and Marklund SL: 10-fold increase in human plasma extracellular superoxide dismutase content caused by a mutation in heparin-binding domain. J Biol Chem 269: 19163-19166, 1994.
28. Horton P, Park KJ, Obayashi T, et al: WoLF PSORT: protein localization predictor. Nucleic Acids Res 35: 585-587, 2007.

29. Connor KM, Hempel N, Nelson KK, et al: Manganese superoxide dismutase enhances the invasive and migratory activity of tumor cells. Cancer Res 67: 10260-10267, 2007.

30. Kim JJ, Chae SW, Hur GC, et al: Manganese superoxide dismutase expression correlates with a poor prognosis in gastric cancer. Pathobiology 70: 353-360, 2003.

31. Janssen AM, Bosman CB, Sier CF, et al: Superoxide dismutases in relation to the overall survival of colorectal cancer patients. Br J Cancer 78: 1051-1057, 1998.

32. Yokoe H, Nomura H, Yamano Y, et al: Characterization of intracellular superoxide dismutase alterations in premalignant and malignant lesions of the oral cavity: correlation with lymph node metastasis. J Cancer Res Clin Oncol 135: 1625-1633, 2009. 